\title{
A NORTHCOTT TYPE INEQUALITY FOR BUCHSBAUM-RIM COEFFICIENTS
}

\author{
R. BALAKRISHNAN AND A.V. JAYANTHAN
}

\begin{abstract}
In 1960, Northcott [13] proved that, if $e_{0}(I)$ and $e_{1}(I)$ denote the 0th and first Hilbert-Samuel coefficients of an m-primary ideal $I$ in a Cohen-Macaulay local ring $(R, \mathfrak{m})$, then $e_{0}(I)-e_{1}(I) \leq \ell(R / I)$. In this article, we study an analogue of this inequality for Buchsbaum-Rim coefficients. We prove that, if $(R, \mathfrak{m})$ is a two dimensional Cohen-Macaulay local ring and $M$ is a finitely generated $R$ module contained in a free module $F$ with finite co-length, then $\operatorname{br}_{0}(M)-\operatorname{br}_{1}(M) \leq \ell(F / M)$, where $\operatorname{br}_{0}(M)$ and $\operatorname{br}_{1}(M)$ denote 0th and 1st Buchsbaum-Rim coefficients, respectively.
\end{abstract}

1. Introduction. Let $(R, \mathfrak{m})$ be a Noetherian local ring of dimension $d>0$. Let $M \subset F=R^{r}$ be a finitely generated $R$-module such that $\ell(F / M)<\infty$, where $\ell(-)$ denotes the length function. Let

$$
\mathcal{S}(F)=\bigoplus_{n \geq 0} \mathcal{S}_{n}(F)
$$

denote the symmetric algebra of $F$ and

$$
\mathcal{R}(M)=\bigoplus_{n \geq 0} \mathcal{R}_{n}(M)
$$

the Rees algebra of $M$, which is image of the natural map from the symmetric algebra of $M$ to the symmetric algebra of $F$.

Generalizing the notion of the Hilbert-Samuel function, Buchsbaum and Rim studied the function

$$
B F(n)=\ell\left(\mathcal{S}_{n}(F) / \mathcal{R}_{n}(M)\right) \quad \text { for } n \in \mathbb{N} .
$$

2010 AMS Mathematics subject classification. Primary 13A30, 13D40.

Keywords and phrases. Buchsbaum-Rim function, Buchsbaum-Rim polynomial, Northcott inequality, Rees algebra of modules.

The first author was supported by the Council of Scientific and Industrial Research (CSIR), India.

Received by the editors on July 21, 2014. 
In [3], it is proved that $B F(n)$ is given by a polynomial of degree $d+r-1$ for $n \gg 0$, i.e., there exists a polynomial $B P(x) \in \mathbb{Q}[x]$ such that $B F(n)=B P(n)$ for $n \gg 0$. The function $B F(n)$ is called the Buchsbaum-Rim function of $M$ with respect to $F$ and the polynomial $B P(n)$ is called the corresponding Buchsbaum-Rim polynomial. Following the notation used for the Hilbert-Samuel polynomial, the Buchsbaum-Rim polynomial is written as:

$$
B P_{M}(n)=\sum_{i=0}^{d+r-1}(-1)^{i} \operatorname{br}_{i}(M)\left(\begin{array}{c}
n+d+r-i-2 \\
d+r-i-1
\end{array}\right) .
$$

The coefficients $\operatorname{br}_{i}(M)$ for $i=0, \ldots, d+r-1$, are known as BuchsbaumRim coefficients.

When $r=1$, set $M=I$, an $\mathfrak{m}$-primary ideal in $R$. In this case, the Buchsbaum-Rim polynomial coincides with the usual Hilbert-Samuel polynomial, and its coefficients will be denoted by $e_{i}(I)$, called the Hilbert-Samuel coefficients. While the Hilbert-Samuel coefficients are very well-studied objects and the relationship of their properties with the properties of the ideal and the corresponding blowup algebras are well known, there is a dearth of results in this direction on BuchsbaumRim coefficients. Northcott proved the following.

Theorem 1.1 ([13, Theorems 1, 3]). Let $(R, \mathfrak{m})$ be a Cohen-Macaulay local ring of dimension $d>0$ with infinite residue field, and let $I$ be an $\mathfrak{m}$-primary ideal. Then:

(i) $e_{0}(I)-e_{1}(I) \leq \ell(R / I)$.

(ii) $e_{1}(I) \geq 0$, and the equality holds if and only if $I$ is generated by $d$ elements, i.e., I is a parameter ideal.

Huneke and Ooishi independently studied the equality in Theorem 1.1 (i):

Theorem $1.2([\mathbf{6}, \mathbf{1 4}])$. Let $(R, \mathfrak{m})$ be a Cohen-Macaulay local ring of dimension $d>0$, and let $I$ be an $\mathfrak{m}$-primary ideal of $R$. Then $e_{0}(I)-e_{1}(I)=\ell(R / I)$ if and only if there exists a minimal reduction $J \subset I$ such that $I^{2}=J I$. 
In [2], Brennan, Ulrich and Vasconcelos proved that Theorem 1.1 (ii) generalizes to the Buchsbaum-Rim coefficient. If $(R, \mathfrak{m})$ is a CohenMacaulay ring, then $\operatorname{br}_{1}(M)$ is non-negative and $\operatorname{br}_{1}(M)$ vanishes if and only if $M$ is a parameter module. In [5], Hayasaka and Hyry studied the Buchsbaum-Rim function of a parameter module $N$ over a Noetherian local ring, and they proved that $\operatorname{br}_{1}(N) \leq 0$ and equality holds if and only if the ring is Cohen-Macaulay.

Motivated by Theorems 1.1 and 1.2, we ask the following.

Question 1.3. Let $(R, \mathfrak{m})$ be a Cohen-Macaulay local ring of dimension $d>0, F$ a free module of rank $r$ and $M$ a submodule such that $\ell(F / M)<\infty$. Then is the inequality $\operatorname{br}_{0}(M)-\operatorname{br}_{1}(M) \leq \ell(F / M)$ true? Is it true that the equality holds if and only if the reduction number of $M$ with respect to a minimal reduction is at most one?

In this article, we prove inequality in the case $\operatorname{dim} R=2$ and show that the module having reduction number 1 is a sufficient condition for equality. We now give a short description of the paper.

In Section 2, we begin with an example to show that the Northcott type inequality does not hold true for Buchsbaum-Rim coefficients if $\operatorname{dim} R=1$. We then consider the cases $\operatorname{dim} R=d \geq 2$ and $M=I_{1} \oplus \cdots \oplus I_{r} \subset R^{r}$, where $I_{i}$ 's are $\mathfrak{m}$-primary ideals in $R$. When the Rees algebra $\mathcal{R}(M)$ is Cohen-Macaulay, we obtain an expression for the Buchsbaum-Rim coefficients $\operatorname{br}_{0}(M)$ and $\operatorname{br}_{1}(M)$ in terms of the mixed multiplicities of the ideals $I_{1}, \ldots, I_{r}$ and derive that, if $d=2$ and $r=2$, we have the equality $\operatorname{br}_{0}(M)-\operatorname{br}_{1}(M)=\ell(F / M)$. We also prove that if $\operatorname{dim} R=2$ and $M$ is an $R$-submodule of $F=R^{r}$ with reduction number of $M$ being one, then $\operatorname{br}_{0}(M)-\operatorname{br}_{1}(M)=\ell(F / M)$.

In Section 3, we define an analogue of the Sally module of a module with respect to a reduction. We obtain an expression for the Hilbert polynomial of the Sally module using the Buchsbaum-Rim coefficients and derive the inequality $\operatorname{br}_{0}(M)-\mathrm{br}_{1}(M) \leq \ell(F / M)$ when $\operatorname{dim} R=2$. We also prove that if $\operatorname{red}(M)=1$, then the equality holds, Theorem 3.3.

In Section 4, we study the problem for modules which are direct sums of several copies of an $\mathfrak{m}$-primary ideal. Let $(R, \mathfrak{m})$ be a CohenMacaulay local ring of dimension $d \geq 2$ and $I$ an $\mathfrak{m}$-primary ideal. 
Let

$$
M=I \oplus \cdots \oplus I \quad(r \text {-times, } r \geq 1) .
$$

Then $\operatorname{br}_{0}(M)-\operatorname{br}_{1}(M) \leq \ell(F / M)$, Theorem 4.1. We also prove that, in dimension 2 , the equality holds if and only if $\operatorname{red}(M)=1$, Corollary 4.3. In addition, we compute some examples to illustrate the Northcott inequality.

2. Reduction number one. In this section, we obtain certain sufficient conditions for the equality $\operatorname{br}_{0}(M)-\operatorname{br}_{1}(M)=\ell(F / M)$. We begin by recalling some basic terminology which is essential for studying the Buchsbaum-Rim polynomial. Let $M \subseteq F=R^{r}$ be such that $\ell(F / M)<\infty$. Let $N$ be a submodule of $M$. We say that $N$ is a reduction of $M$ if the Rees algebra $\mathcal{R}(M)$ is integral over the $R$ subalgebra $\mathcal{R}(N)$. Equivalently, this condition is expressed as

$$
\mathcal{R}_{n+1}(M)=N \mathcal{R}_{n}(M) \text { for } n \gg 0,
$$

where the multiplication is done as $R$-submodules of $\mathcal{R}(M)$. The least integer $s$ such that $\mathcal{R}_{s+1}(M)=N \mathcal{R}_{s}(M)$ is called the reduction number of $M$ with respect to $N$, and denoted as $\operatorname{red}_{N}(M)$. The reduction number of the module $M$, denoted $\operatorname{red}(M)$, is defined as

$$
\operatorname{red}(M)=\min \left\{\operatorname{red}_{N}(M): N \text { is a minimal reduction of } \mathrm{M}\right\} .
$$

If $N$ is a submodule of $F$ generated by $d+r-1$ elements such that $\ell(F / N)<\infty$, then $N$ is said to be a parameter module. It was proved [2] that, if $\ell(F / M)<\infty$, then there exists a minimal reduction generated by $d+r-1$ elements. For more details on minimal reductions we refer the reader to $[\mathbf{7}, \mathbf{1 7}]$.

In the following example, we show that, for one dimensional CohenMacaulay local rings, the Northcott type inequality does not hold for Buchsbaum-Rim coefficients.

Example 2.1. Let $R=k[[X, Y]] /\left(X^{2}\right)$ and $I=(x, y)$, where $x=\bar{X}$ and $y=\bar{Y}$, and $k$ is a field. Then $R$ is a one-dimensional CohenMacaulay local ring. It can be seen that

$$
\ell\left(R / I^{n}\right)=\ell\left(k[[X, Y]] /\left(X^{2},(X, Y)^{n}\right)\right)=2 n-1 .
$$

Therefore, $e_{0}=2$ and $e_{1}=1$. 
Let $F=R \oplus R$ and $M=I \oplus I$. Then it follows from [15, Theorem 2.5.2] that the Buchsbaum-Rim polynomial of $M$ is given by:

$$
\begin{aligned}
B P(n) & =\left[e_{0} n-e_{1}\right]\left(\begin{array}{c}
n+1 \\
1
\end{array}\right) \\
& =2 e_{0}\left(\begin{array}{c}
n+1 \\
2
\end{array}\right)-e_{1}\left(\begin{array}{l}
n \\
1
\end{array}\right)-e_{1} \\
& =4\left(\begin{array}{c}
n+1 \\
2
\end{array}\right)-\left(\begin{array}{l}
n \\
1
\end{array}\right)-1 .
\end{aligned}
$$

Hence, we have $\operatorname{br}_{0}(M)=4$ and $\operatorname{br}_{1}(M)=1$. Therefore,

$$
\mathrm{br}_{0}-\mathrm{br}_{1}=3>2=\ell(F / M) \text {. }
$$

Now we study the Buchsbaum-Rim polynomial of a special class of modules, namely, a direct sum of $\mathfrak{m}$-primary ideals in a CohenMacaulay local ring. Let $(R, \mathfrak{m})$ be a $d$-dimensional Noetherian local ring and $\mathbf{I}=I_{1}, \ldots, I_{r}$ a sequence of $\mathfrak{m}$-primary ideals. For $\underline{u}=$ $\left(u_{1}, \ldots, u_{r}\right) \in \mathbb{N}^{r}$, let $\mathbf{I}^{\underline{u}}=I_{1}^{u_{1}} \cdots I_{r}^{u_{r}}$. Then $\ell\left(R / \mathbf{I}^{\underline{u}}\right)$ is given by a polynomial $P(\underline{u})$ in $r$ variables of total degree $d$ for $u_{i} \gg 0$ for each $i$ [1]. Write the Bhattacharya polynomial of $\mathbf{I}$ as

$$
P_{\mathbf{I}}(\underline{u})=\sum_{\substack{\alpha \in \mathbb{N}^{r} \\
|\alpha| \leq d}} e_{\alpha}(\mathbf{I})\left(\begin{array}{c}
u_{1} \\
\alpha_{1}
\end{array}\right) \cdots\left(\begin{array}{c}
u_{r} \\
\alpha_{r}
\end{array}\right) .
$$

Here, $e_{\alpha}(\mathbf{I})$ with $|\alpha|=d$ are known as the mixed multiplicities of $I_{1}, \ldots, I_{r}$.

For $i=0, \ldots, d$, set $E_{i}=\sum_{\alpha \in \mathbb{N}^{r},|\alpha|=i} e_{\alpha}(\mathbf{I})$. Below, we obtain an expression for the Buchsbaum-Rim multiplicity and the first BuchsbaumRim coefficient in terms of the Bhattacharya coefficients.

Proposition 2.2. Let $(R, \mathfrak{m})$ be the d-dimensional Cohen-Macaulay local ring, $I_{1}, \ldots, I_{r}$ the $\mathfrak{m}$-primary ideals and $M=I_{1} \oplus \cdots \oplus I_{r} \subset R^{r}$. If

$$
\ell\left(R / I^{\underline{u}}\right)=P_{\boldsymbol{I}}(\underline{u}) \quad \text { for all } \underline{u} \in \mathbb{N}^{r},
$$

then $\operatorname{br}_{0}(M)=E_{d}$ and $\operatorname{br}_{1}(M)=(d-1) E_{d}-E_{d-1}$. 
Proof. Let $B P(n)$ denote the Buchsbaum-Rim polynomial corresponding to the function $B F(n)=\ell\left(\mathcal{S}_{n}(F) / \mathcal{R}_{n}(M)\right)$. First note that

$$
\mathcal{S}(F) \cong R\left[t_{1}, \ldots, t_{r}\right] \text { and } \mathcal{R}(M) \cong R\left[I_{1} t_{1}, \ldots, I_{r} t_{r}\right]
$$

where $t_{1}, \ldots, t_{r}$ are indeterminates over $R$. Then

$$
B F(n)=\sum_{\substack{\underline{u} \in \mathbb{N}^{r} \\|\underline{u}|=n}} \ell\left(R / \mathbf{I}^{\underline{u}}\right) .
$$

Hence, for all $n \in \mathbb{N}$, we have

$$
\begin{aligned}
& B P(n)=B F(n)=\sum_{\substack{u \in \mathbb{N} r \\
|\underline{u}|=n}} P_{\mathbf{I}}(\underline{u}) \\
& =\sum_{\substack{u \in \mathbb{N}^{r} \\
|\underline{u}|=n}} \sum_{\substack{\alpha \in \mathbb{N}^{r} \\
|\underline{\alpha}| \leq d}} e_{\underline{\alpha}}(\mathbf{I})\left(\begin{array}{c}
u_{1} \\
\alpha_{1}
\end{array}\right) \cdots\left(\begin{array}{c}
u_{r} \\
\alpha_{r}
\end{array}\right) \\
& =\sum_{\substack{\alpha \in \mathbb{N}^{r} \\
|\underline{\alpha}| \leq d}} e_{\underline{\alpha}}(\mathbf{I}) \sum_{\substack{u \in \mathbb{N}^{r} \\
|\underline{u}|=n}}\left(\begin{array}{c}
u_{1} \\
\alpha_{1}
\end{array}\right) \cdots\left(\begin{array}{c}
u_{r} \\
\alpha_{r}
\end{array}\right) \\
& =\sum_{\substack{\underline{\alpha} \in \mathbb{N}^{r} \\
|\underline{\alpha}| \leq d}} e_{\underline{\alpha}}(\mathbf{I})\left(\begin{array}{c}
n+r-1 \\
|\underline{\alpha}|+r-1
\end{array}\right) \\
& =E_{d}\left(\begin{array}{l}
n+r-1 \\
d+r-1
\end{array}\right)+E_{d-1}\left(\begin{array}{l}
n+r-1 \\
d+r-2
\end{array}\right)+\cdots .
\end{aligned}
$$

By using Pascal's identity repeatedly, we observe that

$$
\begin{aligned}
\left(\begin{array}{c}
n+r-1 \\
d+r-1
\end{array}\right)= & \left(\begin{array}{c}
n+d+r-2 \\
d+r-1
\end{array}\right) \\
& -\left[\left(\begin{array}{c}
n+d+r-3 \\
d+r-2
\end{array}\right)+\cdots+\left(\begin{array}{c}
n+r-1 \\
d+r-2
\end{array}\right)\right]
\end{aligned}
$$

Hence,

$$
B P(n)=E_{d}\left(\begin{array}{c}
n+d+r-2 \\
d+r-1
\end{array}\right)+\left[E_{d-1}-(d-1) E_{d}\right]\left(\begin{array}{c}
n+d+r-3 \\
d+r-2
\end{array}\right)+\cdots .
$$

It follows that $\operatorname{br}_{0}(M)=E_{d}$ and $\operatorname{br}_{1}(M)=(d-1) E_{d}-E_{d-1}$.

Note that, if $\mathcal{R}(M)$ is Cohen-Macaulay, then by [9, Theorem 6.1], $\ell\left(R / \mathbf{I}^{\underline{u}}\right)=P_{\mathbf{I}}(\underline{u})$ for all $\underline{u} \in \mathbb{N}^{r}$, and hence, $B F(n)=B P(n)$ for all 
$n \geq 0$. As a consequence, we obtain the equality $\operatorname{br}_{0}(M)-\operatorname{br}_{1}(M)=$ $\ell(F / M)$ :

Corollary 2.3. Let $(R, \mathfrak{m})$ be a two dimensional Cohen-Macaulay local ring with infinite residue field. Let $I$ and $J$ be $\mathfrak{m}$-primary ideals in $R$ and $M=I \oplus J \subset R \oplus R$. If $\mathcal{R}(M)$ is Cohen-Macaulay, then $\operatorname{br}_{0}(M)-\operatorname{br}_{1}(M)=\ell(F / M)$.

Proof. By applying Proposition 2.2 with $d=2$ and $r=2$, we get $\operatorname{br}_{0}(M)-\operatorname{br}_{1}(M)=E_{2}-\left(E_{2}-E_{1}\right)=E_{1}=e_{10}+e_{01}$. Since $\mathcal{R}(M)$ is Cohen-Macaulay, it follows from [10, Theorem 6.3] that $e_{10}=\ell(R / I)$ and $e_{01}=\ell(R / J)$. Therefore,

$$
\operatorname{br}_{0}(M)-\operatorname{br}_{1}(M)=\ell(R / I)+\ell(R / J)=\ell(F / M) .
$$

Note that Corollary 2.3 can also be derived from Theorem 2.10. We have provided the above proof as it is independent and involves a different technique.

Remark 2.4. Let $(R, \mathfrak{m})$ be a two dimensional Cohen-Macaulay local ring, let $I_{1}, \ldots, I_{r}$ be $\mathfrak{m}$-primary ideals, and let $M=I_{1} \oplus \cdots \oplus I_{r}$. Let $\operatorname{jr}\left(I_{i} \mid I_{j}\right)$ denote the joint reduction number of $I_{i}$ and $I_{j}$ (we refer the reader to $[\mathbf{8}, \mathbf{1 8}]$ for the definition and some basic results concerning joint reductions). It is proved [16, Corollary 4.5] that, if $\mathrm{jr}\left(I_{i} \mid I_{j}\right)=0$ for any $i, j \in\{1, \ldots, r\}$, then $\mathcal{R}(M)$ is Cohen-Macaulay. We would like to observe here that the converse is also true. Suppose $\mathcal{R}(M)$ is Cohen-Macaulay. Then a modification of [12, Theorem 6.1] gives that $\mathcal{R}\left(I_{i_{1}} \oplus \cdots \oplus I_{i_{s}}\right)$ is Cohen-Macaulay for any $\left\{i_{1}, \ldots, i_{s}\right\} \subset\{1, \ldots, r\}$. In particular, $\mathcal{R}\left(I_{i}\right)$ is Cohen-Macaulay for each $i=1, \ldots, r$ and $\mathcal{R}\left(I_{i} \oplus I_{j}\right)$ is Cohen-Macaulay for $\{i, j\} \subset\{1, \ldots, r\}$. This implies that $\operatorname{jr}\left(I_{i} \mid I_{j}\right)=0$ for any $1 \leq i, j \leq r$.

In the following example, we compute the Buchsbaum-Rim coefficients.

Example 2.5. Let $R=k[[X, Y]], I=\mathfrak{m}=(X, Y)$ and $J=\left(X^{2}, Y\right)$. Then $\operatorname{red}(I)=\operatorname{red}(J)=0$. Also, $(Y) I+(X) J=I J$ implying $\operatorname{jr}(I \mid J)=0$ so that the Rees algebra $R(I, J) \cong \mathcal{R}(I \oplus J)$ is CohenMacaulay [10, Theorem 6.3]. Set $F=R \oplus R$ and $M=I \oplus J$. Therefore, 
we have $B F(n)=B P(n)$ for all $n$. Using any of the computational commutative algebra packages, it can be seen that

$$
\begin{aligned}
& \ell\left(\mathcal{S}_{1}(F) / \mathcal{R}_{1}(M)\right)=3, \\
& \ell\left(\mathcal{S}_{2}(F) / \mathcal{R}_{2}(M)\right)=13, \\
& \ell\left(\mathcal{S}_{3}(F) / \mathcal{R}_{3}(M)\right)=34, \\
& \ell\left(\mathcal{S}_{4}(F) / \mathcal{R}_{4}(M)\right)=70 .
\end{aligned}
$$

In turn, we get the Buchsbaum-Rim polynomial as

$$
B P(n)=4\left(\begin{array}{c}
n+2 \\
3
\end{array}\right)-1\left(\begin{array}{c}
n+1 \\
2
\end{array}\right) .
$$

Hence, $\operatorname{br}_{0}(M)-\operatorname{br}_{1}(M)=4-1=3=\ell(F / M)$.

Katz and Kodiyalam studied the Cohen-Macaulayness of the Rees algebra of modules over two-dimensional regular local rings. They proved:

Theorem 2.6 ([11, Corollary 4.2]). Let $(R, \mathfrak{m})$ be a two-dimensional regular local ring, and let $M$ be a finitely generated torsion free $R$ module. Then the following are equivalent:

(i) $N M=\mathcal{R}_{2}(M)$ for every minimal reduction $N \subset M$;

(ii) The Rees algebra $\mathcal{R}(M)$ is Cohen-Macaulay;

(iii) $\ell\left(\mathcal{S}_{n+1}(F) / \mathcal{R}_{n+1}(M)\right)=\operatorname{br}_{0}(M)\left(\begin{array}{c}n+r+1 \\ r+1\end{array}\right)-\ell(M / N)\left(\begin{array}{c}n+r \\ r\end{array}\right)$ for all $n \geq 0$ and every minimal reduction $N \subset M$.

Since $N$ is a parameter module and a minimal reduction of $M$, $\operatorname{br}_{0}(M)=\operatorname{br}_{0}(N)=\ell(F / N),[2$, Theorem 3.1]. Hence, in this case, $\operatorname{br}_{0}(M)-\operatorname{br}_{1}(M)=\ell(F / N)-\ell(M / N)=\ell(F / M)$. Simis, Ulrich and Vasconcelos proved that, if $(R, \mathfrak{m})$ is a two dimensional Cohen-Macaulay local ring and $M \subset F=R^{r}$ is a module with $\ell(F / M)<\infty$, then $\mathcal{R}(M)$ is Cohen-Macaulay if and only if $\operatorname{red}(M) \leq 1$ [16, Proposition 4.4]. By adopting the proof of Katz and Kodiyalam, we prove (i) implies (iii) of Theorem 2.6 in the case of two-dimensional Cohen-Macaulay rings. Although the proof works along the same lines, the two isomorphisms used in the proof are justified by a result of Hayasaka and Hyry. We recall the result from [4]. For an $R$-module $M$, 
let $\widetilde{M}$ denote the matrix whose columns correspond to the generators of $M$ with respect to a fixed basis of $F$. The matrix $\widetilde{M}$ is said to be perfect if the 0 th fitting ideal of $M$ is a proper ideal with maximal grade.

Theorem 2.7 ([4, Theorem 4.4]). Let $R$ be a Noetherian ring and $F$ an $R$-free module of rank $r>0$. Let $M$ be a submodule of $F$ such that $\widetilde{M}$ is a perfect matrix of size $r \times(r+1)$. Then the natural surjective homomorphism

$$
\phi_{1}:(F / M)\left[Y_{1}, \ldots, Y_{r+1}\right] \longrightarrow G_{1}(M)
$$

is an isomorphism, where $G_{1}(M)=F \mathcal{R}(M) / \mathcal{R}(M)^{+}$.

In particular, the $R$-module $F \mathcal{R}_{n}(M) / \mathcal{R}_{n+1}(M)$ is a direct sum of $\left(\begin{array}{c}n+r \\ r\end{array}\right)$ copies of $F / M$.

Remark 2.8. It is known that, if $M$ is a parameter module, then the matrix $\widetilde{M}$ is perfect [4]. So in particular, when the $\operatorname{ring} R$ is a two dimensional Cohen-Macaulay local ring and $M$ is a parameter module, Theorem 2.7 is true [4, Corollary 4.5].

Lemma 2.9. Let $(R, \mathfrak{m})$ be a two dimensional Cohen-Macaulay local ring with infinite residue field and $M \subset F=R^{r}$ a finitely generated $R$-module with $\ell(F / M)<\infty$. Let $N \subset M$ be a minimal reduction generated by $\left\{c_{1}, \ldots, c_{r+1}\right\}$. If

$$
k=\left(\begin{array}{c}
n+r \\
r
\end{array}\right) \quad \text { and } \quad \phi: F^{k} \longrightarrow F \mathcal{R}_{n}(N)
$$

is the surjective $R$-module homomorphism defined by

$$
\phi\left(f_{1}, \ldots, f_{k}\right)=\sum_{\substack{i=1 \\ i_{1}+\cdots+i_{r+1}=n}}^{k} f_{i} c_{1}^{i_{1}} c_{2}^{i_{2}} \cdots c_{r+1}^{i_{r+1}},
$$

then the corresponding induced maps

$$
\phi_{1}:\left(\frac{F}{N}\right)^{k} \longrightarrow \frac{F \mathcal{R}_{n}(N)}{\mathcal{R}_{n+1}(N)} \quad \text { and } \quad \phi_{2}:\left(\frac{F}{M}\right)^{k} \longrightarrow \frac{F \mathcal{R}_{n}(N)}{M \mathcal{R}_{n}(N)}
$$

are isomorphisms. 
Proof. It follows from Remark 2.8 that $\phi_{1}$ is an isomorphism. Surjectivity of $\phi_{2}$ is clear. For an element $f \in F$, let $\bar{f}$ denote its image in $F / M$ and $\tilde{f}$ its image in $F / N$. Suppose $\phi_{2}\left(\overline{f_{1}}, \ldots, \overline{f_{k}}\right)=0$. This implies

$$
\sum_{\substack{i_{1}+\cdots+i_{r+1}=n \\ i=1}}^{k} f_{i} c_{1}^{i_{1}} c_{2}^{i_{2}} \cdots c_{r+1}^{i_{r+1}}=\sum_{\substack{i_{1}+\cdots+i_{r+1}=n \\ i=1}}^{k} g_{i} c_{1}^{i_{1}} c_{2}^{i_{2}} \cdots c_{r+1}^{i_{r+1}}
$$

for some $g_{i} \in M$. This implies that $\phi_{1}\left(\widetilde{f_{1}-g_{1}}, \ldots, \widetilde{f_{k}-g_{k}}\right)=0$. Since $\phi_{1}$ is injective, it follows that $f_{i}-g_{i} \in N \subset M$ for all $i=1, \ldots, k$. Hence, $f_{i} \in M$ for $i=1, \ldots, k$.

Now we prove (i) implies (iii) in Theorem 2.6 for two dimensional Cohen-Macaulay rings.

Theorem 2.10. Let $(R, \mathfrak{m})$ be a two dimensional Cohen-Macaulay local ring with infinite residue field and $M \subset F=R^{r}$ a finitely generated $R$-module with $\ell(F / M)<\infty$. If $\operatorname{red}_{N}(M)=1$ for a minimal reduction $N \subset M$, then, for all $n \geq 0$,

$$
\ell\left(\mathcal{S}_{n+1}(F) / \mathcal{R}_{n+1}(M)\right)=\ell(F / N)\left(\begin{array}{c}
n+r+1 \\
r+1
\end{array}\right)-\ell(M / N)\left(\begin{array}{c}
n+r \\
r
\end{array}\right) .
$$

In particular, if for any minimal reduction $N$ of $M \operatorname{red}_{N}(M)=1$, then $\operatorname{br}_{0}(M)-\operatorname{br}_{1}(M)=\ell(F / M)$ and $\operatorname{br}_{i}(M)=0$ for all $i=2, \ldots, r+1$.

Proof. Since $\operatorname{red}_{N}(M)$ is one, we have $\mathcal{R}_{2}(M)=N \mathcal{R}_{1}(M)$. This implies $\mathcal{R}_{n+1}(M)=N \mathcal{R}_{n}(M)$ for all $n \geq 1$. By induction, one can see that $\mathcal{R}_{n+1}(M)=M \mathcal{R}_{n}(N)$ for all $n \geq 0$. Consider the following short exact sequences of $R$-modules with natural maps

$$
\begin{aligned}
0 \longrightarrow \frac{\mathcal{S}_{1}(F) \mathcal{R}_{n}(N)}{\mathcal{R}_{1}(M) \mathcal{R}_{n}(N)} \longrightarrow \frac{\mathcal{S}_{n+1}(F)}{\mathcal{R}_{n+1}(M)} \longrightarrow \frac{\mathcal{S}_{n+1}(F)}{\mathcal{S}_{1}(F) \mathcal{R}_{n}(N)} \longrightarrow 0, \\
0 \longrightarrow \frac{\mathcal{S}_{1}(F) \mathcal{R}_{n}(N)}{\mathcal{R}_{n+1}(N)} \longrightarrow \frac{\mathcal{S}_{n+1}(F)}{\mathcal{R}_{n+1}(N)} \longrightarrow \frac{\mathcal{S}_{n+1}(F)}{\mathcal{S}_{1}(F) \mathcal{R}_{n}(N)} \longrightarrow 0
\end{aligned}
$$

By additivity of the length function on the short exact sequences, we get

$\ell\left(\frac{\mathcal{S}_{n+1}(F)}{\mathcal{R}_{n+1}(M)}\right)=\ell\left(\frac{\mathcal{S}_{n+1}(F)}{\mathcal{R}_{n+1}(N)}\right)+\ell\left(\frac{\mathcal{S}_{1}(F) \mathcal{R}_{n}(N)}{\mathcal{R}_{1}(M) \mathcal{R}_{n}(N)}\right)-\ell\left(\frac{\mathcal{S}_{1}(F) \mathcal{R}_{n}(N)}{\mathcal{R}_{n+1}(N)}\right)$. 
Let $k=\left(\begin{array}{c}n+r \\ r\end{array}\right)$. By Lemma 2.9,

$$
\left(\frac{F}{M}\right)^{k} \cong \frac{F \mathcal{R}_{n}(N)}{M \mathcal{R}_{n}(N)} \quad \text { and } \quad\left(\frac{F}{N}\right)^{k} \cong \frac{F \mathcal{R}_{n}(N)}{\mathcal{R}_{n+1}(N)}
$$

Hence,

$$
\ell\left(\frac{F \mathcal{R}_{n}(N)}{M \mathcal{R}_{n}(N)}\right)=\ell(F / M)\left(\begin{array}{c}
n+r \\
r
\end{array}\right)
$$

and

$$
\ell\left(\frac{F \mathcal{R}_{n}(N)}{\mathcal{R}_{n+1}(N)}\right)=\ell(F / N)\left(\begin{array}{c}
n+r \\
r
\end{array}\right)
$$

Since $N$ is a parameter module, by [2, Theorem 3.4],

$$
\ell\left(\mathcal{S}_{n+1}(F) / \mathcal{R}_{n+1}(N)\right)=\operatorname{br}_{0}(N)\left(\begin{array}{c}
n+r+1 \\
r+1
\end{array}\right)=\operatorname{br}_{0}(M)\left(\begin{array}{c}
n+r+1 \\
r+1
\end{array}\right) .
$$

Therefore,

$$
\begin{aligned}
\ell\left(\frac{\mathcal{S}_{n+1}(F)}{\mathcal{R}_{n+1}(M)}\right)= & \operatorname{br}_{0}(M)\left(\begin{array}{c}
n+r+1 \\
r+1
\end{array}\right) \\
& +[\ell(F / M)-\ell(F / N)]\left(\begin{array}{c}
n+r \\
r
\end{array}\right) \\
= & \operatorname{br}_{0}(M)\left(\begin{array}{c}
n+r+1 \\
r+1
\end{array}\right)-\ell(M / N)\left(\begin{array}{c}
n+r \\
r
\end{array}\right) \\
= & \ell(F / N)\left(\begin{array}{c}
n+r+1 \\
r+1
\end{array}\right)-\ell(M / N)\left(\begin{array}{c}
n+r \\
r
\end{array}\right) .
\end{aligned}
$$

The second assertion now follows from the above equality.

The main hurdle in proving a $d$-dimensional version of Theorem 2.10 is in generalizing Theorem 2.7, which is not known for modules $M$ with $\widetilde{M}$ being a perfect matrix of size $r \times(d+r-1)$, where $d=\operatorname{dim} R$.

3. Main result. In this section, we prove an analogue of the Northcott inequality for submodules of free modules over two dimensional Cohen-Macaulay rings, which have finite co-length. Vasconcelos introduced the notion of Sally modules $S_{J}(I)$, where $I$ is an ideal with a reduction $J$, to study the interplay between the depth properties of 
blowup algebras and the properties of the Hilbert-Samuel coefficients. The Sally module $S_{J}(I)$ of $I$ with respect to $J$ is the $\mathcal{R}(J)$-module defined by the following short exact sequence

$$
0 \longrightarrow I \mathcal{R}(J) \longrightarrow I \mathcal{R}(I) \longrightarrow S_{J}(I):=\oplus_{n \geq 0} I^{n+1} / I J^{n} \longrightarrow 0 .
$$

We refer the reader to [17] for basic properties of Sally modules. This definition can be extended to inclusion of graded algebras, [17]. As we have $\oplus_{n} \mathcal{R}_{n}(N) \subseteq \oplus_{n} \mathcal{R}_{n}(M)$ for any reduction $N$ of $M$, we define the Sally module in an analogous manner:

Definition 3.1. Let $(R, \mathfrak{m})$ be a Noetherian local ring and $M \subset$ $F=R^{r}$ a finitely generated $R$-module. Let $N \subset M$ be a $R$ submodule. Then the Sally module of $M$ with respect to $N$ is defined as $S_{N}(M):=\oplus_{n \geq 1} \mathcal{R}_{n+1}(M) / M \mathcal{R}_{n}(N)$.

We note that $S_{N}(M)$ is zero if and only if $\operatorname{red}_{N}(M)$ is at most one. Note also that $\mathcal{R}(N)$ is a finitely generated standard graded algebra over $R$ and $S_{N}(M)$ is a finitely generated module over $\mathcal{R}(N)$. Suppose $M \subset F=R^{r}$ is such that $\ell(F / M)<\infty$ and $N$ is a minimal reduction of $M$. Then the Hilbert function theory for graded modules says that the Hilbert function, $H(n)=\ell_{R} \mathcal{R}_{n+1}(M) / M \mathcal{R}_{n}(N)$, is given by a polynomial for $n \gg 0$ of degree equal to the dimension of $S_{N}(M)$. Since $\mathfrak{m} \mathcal{R}(N) \subset \mathfrak{p}$ for all $\mathfrak{p} \in \operatorname{Ass}\left(S_{N}(M)\right)$, it follows that $\operatorname{dim} S_{N}(M) \leq d+r-1$. In the following theorem, we relate the Hilbert function of $S_{N}(M)$ and the Buchsbaum-Rim function of module $M$ in the two dimensional Cohen-Macaulay ring. As a consequence, we obtain the Northcott inequality. The proof is analogous to the corresponding results in [17, subsection 2.1.2].

Theorem 3.2. Let $(R, \mathfrak{m})$ be a Cohen-Macaulay local ring of dimension 2 with infinite residue field and $M \subseteq F=R^{r}$ with $\ell(F / M)<\infty$. Let the Buchsbaum-Rim polynomial corresponding to the BuchsbaumRim function $B F(n)=\ell\left(\mathcal{S}_{n}(F) / \mathcal{R}_{n}(M)\right)$ be given by

$$
B P(n)=\operatorname{br}_{0}(M)\left(\begin{array}{c}
n+r \\
r+1
\end{array}\right)-\operatorname{br}_{1}(M)\left(\begin{array}{c}
n+r-1 \\
r
\end{array}\right)+\cdots+(-1)^{r+1} \operatorname{br}_{r+1}(M) .
$$


Suppose $N \subseteq M$ is a minimal reduction and $S=S_{N}(M)$ is the corresponding Sally module. Then for all $n \geq 0$, $B F(n)=\operatorname{br}_{0}(M)\left(\begin{array}{c}n+r \\ r+1\end{array}\right)+\left[\ell(F / M)-\operatorname{br}_{0}(M)\right]\left(\begin{array}{c}n+r-1 \\ r\end{array}\right)-\ell\left(S_{n-1}\right)$.

Proof. Consider the following two short exact sequences of $R$ modules:

$$
\begin{aligned}
0 \longrightarrow \frac{M \mathcal{R}_{n-1}(N)}{\mathcal{R}_{n}(N)} \longrightarrow \frac{\mathcal{R}_{n}(M)}{\mathcal{R}_{n}(N)} \longrightarrow \frac{\mathcal{R}_{n}(M)}{M \mathcal{R}_{n-1}(N)} \longrightarrow 0, \\
0 \longrightarrow \frac{M \mathcal{R}_{n-1}(N)}{\mathcal{R}_{n}(N)} \longrightarrow \frac{F \mathcal{R}_{n-1}(N)}{\mathcal{R}_{n}(N)} \longrightarrow \frac{F \mathcal{R}_{n-1}(N)}{M \mathcal{R}_{n-1}(N)} \longrightarrow 0 .
\end{aligned}
$$

Set $k=\left(\begin{array}{c}n+r \\ r\end{array}\right)$. By Lemma 2.9, it follows that

$$
\ell\left(\frac{F \mathcal{R}_{n}(N)}{M \mathcal{R}_{n}(N)}\right)=\ell(F / M)\left(\begin{array}{c}
n+r \\
r
\end{array}\right)
$$

and

$$
\ell\left(\frac{F \mathcal{R}_{n}(N)}{\mathcal{R}_{n+1}(N)}\right)=\ell(F / N)\left(\begin{array}{c}
n+r \\
r
\end{array}\right)
$$

Therefore, we have

$$
\begin{aligned}
B F(n)= & \ell\left(\frac{\mathcal{S}_{n}(F)}{\mathcal{R}_{n}(M)}\right)=\ell\left(\frac{\mathcal{S}_{n}(F)}{\mathcal{R}_{n}(N)}\right)-\ell\left(\frac{\mathcal{R}_{n}(M)}{\mathcal{R}_{n}(N)}\right) \\
= & \ell\left(\frac{\mathcal{S}_{n}(F)}{\mathcal{R}_{n}(N)}\right)+\ell\left(\frac{F \mathcal{R}_{n-1}(N)}{M \mathcal{R}_{n-1}(N)}\right) \\
& -\ell\left(\frac{F \mathcal{R}_{n-1}(N)}{\mathcal{R}_{n}(N)}\right)-\ell\left(\frac{\mathcal{R}_{n}(M)}{M \mathcal{R}_{n-1}(N)}\right) \\
= & \operatorname{br}_{0}(N)\left(\begin{array}{c}
n+r \\
r+1
\end{array}\right)+\ell\left(\frac{F}{M}\right)\left(\begin{array}{c}
n+r-1 \\
r
\end{array}\right) \\
& -\ell\left(\frac{F}{N}\right)\left(\begin{array}{c}
n+r-1 \\
r
\end{array}\right)-\ell\left(\frac{\mathcal{R}_{n}(M)}{M \mathcal{R}_{n-1}(N)}\right) \\
= & \operatorname{br}_{0}(M)\left(\begin{array}{c}
n+r \\
r+1
\end{array}\right)+\left[\ell(F / M)-\mathrm{br}_{0}(M)\right]\left(\begin{array}{c}
n+r-1 \\
r
\end{array}\right)-\ell\left(S_{n-1}\right) .
\end{aligned}
$$

We now derive the Northcott type inequality for the BuchsbaumRim coefficients in two dimensional Cohen-Macaulay local rings. 
Theorem 3.3. Let $(R, \mathfrak{m})$ be a Cohen-Macaulay local ring of dimension 2 and $M \subset F=R^{r}$ be such that $\ell(F / M)<\infty$. Then $\operatorname{br}_{0}(M)-\operatorname{br}_{1}(M) \leq \ell(F / M)$. If the reduction number of $M$ is at most 1 , then the equality holds.

Proof. Let $B P(n)$ denote the Buchsbaum-Rim polynomial of $M$. Then, by Theorem 3.2 for $n \gg 0$, we get

$$
\begin{aligned}
\ell\left(S_{n-1}\right)= & \operatorname{br}_{0}(M)\left(\begin{array}{c}
n+r \\
r+1
\end{array}\right) \\
& +\left[\ell(F / M)-\operatorname{br}_{0}(M)\right]\left(\begin{array}{c}
n+r-1 \\
r
\end{array}\right)-B P(n) \\
= & {\left[\ell(F / M)-\operatorname{br}_{0}(M)+\operatorname{br}_{1}(M)\right]\left(\begin{array}{c}
n+r-1 \\
r
\end{array}\right) } \\
& -\operatorname{br}_{2}(M)\left(\begin{array}{c}
n+r-2 \\
r-1
\end{array}\right)+\cdots+(-1)^{r} \mathrm{br}_{r+1} .
\end{aligned}
$$

This implies $\ell(F / M)-\mathrm{br}_{0}(M)+\mathrm{br}_{1}(M)$ is non-negative, i.e., $\mathrm{br}_{0}(M)-$ $\operatorname{br}_{1}(M) \leq \ell(F / M)$.

If, for a minimal reduction $N$ of $M, \operatorname{red}_{N}(M) \leq 1$, then $S_{N}(M)=0$, and consequently, $\ell(F / M)-\mathrm{br}_{0}(M)+\mathrm{br}_{1}(M)=0$, i.e., $\mathrm{br}_{0}(M)-$ $\operatorname{br}_{1}(M)=\ell(F / M)$.

4. Direct sum of ideals. In this section, we consider the modules $M$ which are direct sums of several copies of an $\mathfrak{m}$-primary ideal $I$. We explicitly compute $\operatorname{br}_{0}(M)$ and $\operatorname{br}_{1}(M)$ in terms of $e_{0}(I)$ and $e_{1}(I)$. As a consequence, we prove the Northcott inequality in this case. We also prove that, in dimension 2 , the Northcott equality holds if and only if the reduction number is at most 1 .

Theorem 4.1. Let $(R, \mathfrak{m})$ be a Cohen-Macaulay local ring of dimension $d \geq 2$, and let $I$ be an $\mathfrak{m}$-primary ideal. For $r \in \mathbb{N}$, set $F=R^{r}$ and $M=I \oplus \cdots \oplus I$ ( $r$ times $)$. Then $\operatorname{br}_{0}(M)-\operatorname{br}_{1}(M) \leq \ell(F / M)$.

Proof. Let

$$
P_{I}(n)=\sum_{i=0}^{d} e_{i}\left(\begin{array}{c}
n+d-i-1 \\
d-i
\end{array}\right)
$$


be the Hilbert-Samuel polynomial of $I$. Then, by [15, Theorem 2.5.2], the Buchsbaum-Rim polynomial is given by:

$$
\begin{aligned}
B P(n)= & P_{I}(n)\left(\begin{array}{c}
n+r-1 \\
r-1
\end{array}\right) \\
= & {\left[e_{0}\left(\begin{array}{c}
n+d-1 \\
d
\end{array}\right)-e_{1}\left(\begin{array}{c}
n+d-2 \\
d-1
\end{array}\right)+\cdots\right]\left(\begin{array}{c}
n+r-1 \\
r-1
\end{array}\right) } \\
= & e_{0} \frac{(d+r-1) !}{d !(r-1) !}\left(\begin{array}{c}
n+d+r-2 \\
d+r-1
\end{array}\right) \\
& -\left[\begin{array}{c}
\left.e_{0}(d-1) \frac{(d+r-2) !}{d !(r-2) !}+e_{1} \frac{(d+r-2) !}{(d-1) !(r-1) !}\right] \\
\end{array}\right. \\
& \times\left(\begin{array}{c}
n+d+r-3 \\
d+r-2
\end{array}\right)+\cdots .
\end{aligned}
$$

Therefore, $\operatorname{br}_{0}(M)=e_{0}\left(\begin{array}{c}d+r-1 \\ r-1\end{array}\right)$ and

$$
\operatorname{br}_{1}(M)=e_{0}(d-1)\left(\begin{array}{c}
d+r-2 \\
r-2
\end{array}\right)+e_{1}\left(\begin{array}{c}
d+r-2 \\
r-1
\end{array}\right) .
$$

We now split the proof into two cases.

Case 1: $d=2$. In this case, we have $\operatorname{br}_{0}(M)=e_{0}\left(\begin{array}{c}r+1 \\ 2\end{array}\right)$ and $\operatorname{br}_{1}(M)=e_{0}\left(\begin{array}{c}r \\ 2\end{array}\right)+e_{1} r$. Hence,

$$
\operatorname{br}_{0}(M)-\operatorname{br}_{1}(M)=e_{0} r-e_{1} r \leq r \ell(R / I)=\ell(F / M) .
$$

Case 2: $d \geq 3$. Let $r=2$. We then have $\operatorname{br}_{0}(M)=e_{0}(d+1)$ and $\operatorname{br}_{1}(M)=e_{0}(d-1)+e_{1} d$. Therefore,

$\operatorname{br}_{0}(M)-\operatorname{br}_{1}(M)=2 e_{0}-d e_{1}=2\left(e_{0}-e_{1}\right)-(d-2) e_{1} \leq 2 \ell(R / I)=\ell(F / M)$.

Note that, in this case, $\operatorname{br}_{0}(M)-\operatorname{br}_{1}(M)=\ell(F / M)$ if and only if $e_{1}=0$ if and only if $I$ is a parameter ideal.

Now let $r \geq 3$. We then have

$$
\begin{array}{r}
\operatorname{br}_{0}(M)-\operatorname{br}_{1}(M)-\ell(F / M)=e_{0}\left[\left(\begin{array}{c}
d+r-1 \\
r-1
\end{array}\right)-(d-1)\left(\begin{array}{c}
d+r-2 \\
r-2
\end{array}\right)\right] \\
-e_{1}\left(\begin{array}{c}
d+r-2 \\
r-1
\end{array}\right)-r \ell(R / I) .
\end{array}
$$


If $d=3$ and $r=3$, then the above expression becomes

$$
\begin{aligned}
10 e_{0}-8 e_{0}-6 e_{1}-3 \ell(R / I) & =2\left(e_{0}-e_{1}\right)-4 e_{1}-3 \ell(R / I) \\
& \leq-4 e_{1}-\ell(R / I) \leq 0 .
\end{aligned}
$$

Since $(R, \mathfrak{m})$ is Cohen-Macaulay, $e_{1} \geq 0$. Therefore, to prove the Northcott inequality, it is enough to show that

$$
\left[\left(\begin{array}{c}
d+r-1 \\
r-1
\end{array}\right)-(d-1)\left(\begin{array}{c}
d+r-2 \\
r-2
\end{array}\right)\right] e_{0}-r \ell(R / I) \leq 0 .
$$

Considering the coefficient of $e_{0}$ in the above expression, we obtain

$$
\begin{aligned}
\left(\begin{array}{c}
d+r-1 \\
r-1
\end{array}\right)-(d-1)\left(\begin{array}{c}
d+r-2 \\
r-2
\end{array}\right) & =\left(\begin{array}{c}
d+r-2 \\
r-2
\end{array}\right)\left[\frac{d+r-1}{r-1}-(d-1)\right] \\
& =\left(\begin{array}{c}
d+r-2 \\
r-2
\end{array}\right)\left[2-\frac{r-2}{r-1} d\right] .
\end{aligned}
$$

It is a simple verification to see that this expression is non-positive, and hence (4.2) holds, for $d=3 ; r \geq 4$ and $d \geq 4 ; r \geq 3$.

Below, we show that the direct sum of parameter ideal, in rank 2, has reduction number one.

Proposition 4.2. Let $(R, \mathfrak{m})$ be a Cohen-Macaulay local ring of dimension $d \geq 2, I=\left(a_{1}, \ldots, a_{d}\right)$ a parameter ideal and $M=I \oplus I$. Then the submodule $N$ of $M$ generated by the columns of the matrix

$$
\left[\begin{array}{ccccc}
a_{1} & a_{2} & \cdots & a_{d} & 0 \\
0 & a_{1} & \cdots & a_{d-1} & a_{d}
\end{array}\right]
$$

is a minimal reduction of $M$ with $\operatorname{red}_{N}(M)=1$.

Proof. Using the isomorphism $\mathcal{R}(M) \cong R\left[I t_{1}, I t_{2}\right]$, we move all the computations to the bigraded Rees algebra. To prove the assertion, it is enough to show that

$$
\begin{aligned}
& I^{2} t_{1}^{2}+I^{2} t_{1} t_{2}+I^{2} t_{2}^{2} \\
& \quad=\left(a_{1} t_{1}, a_{2} t_{1}+a_{1} t_{2}, \ldots, a_{d} t_{1}+a_{d-1} t_{2}, a_{d} t_{2}\right)\left(I t_{1}+I t_{2}\right) .
\end{aligned}
$$

Set

$$
L=\left(a_{1} t_{1}, a_{2} t_{1}+a_{1} t_{2}, \ldots, a_{d} t_{1}+a_{d-1} t_{2}, a_{d} t_{2}\right)\left(I t_{1}+I t_{2}\right) .
$$


We show that, for any $1 \leq i, j \leq d, a_{i} a_{j} t_{1}^{2}, a_{i} a_{j} t_{1} t_{2}, a_{i} a_{j} t_{2}^{2}$ belong to $L$. First, note that, for all $1 \leq i, j \leq d$, the elements $a_{1} a_{j} t_{1}^{2}, a_{1} a_{j} t_{1} t_{2}, a_{i} a_{d} t_{1} t_{2}, a_{i} a_{d} t_{2}^{2}$ are all in $L$. Consider the following set of equations:

$$
\begin{aligned}
a_{i} a_{j} t_{1}^{2} & =a_{j} t_{1}\left(a_{i} t_{1}+a_{i-1} t_{2}\right)-a_{j} a_{i-1} t_{1} t_{2} \\
a_{j} a_{i-1} t_{1} t_{2} & =a_{j} t_{2}\left(a_{i-1} t_{1}+a_{i-2} t_{2}\right)-a_{j} a_{i-2} t_{2}^{2} \\
a_{j} a_{i-2} t_{2}^{2} & =a_{i-2} t_{2}\left(a_{j+1} t_{1}+a_{j} t_{2}\right)-a_{i-2} a_{j+1} t_{1} t_{2} \\
a_{i-2} a_{j+1} t_{1} t_{2} & =a_{i-2} t_{1}\left(a_{j+2} t_{1}+a_{j+1} t_{2}\right)-a_{i-2} a_{j+2} t_{1}^{2} .
\end{aligned}
$$

Then $a_{i} a_{j} t_{1}^{2} \in L$ if and only if $a_{i-2} a_{j+2} t_{1}^{2} \in L$. If $i=2$, the first equation itself will yield that $a_{i} a_{j} t_{1}^{2} \in L$. If $j=d-1$, then the third equation will yield that $a_{i} a_{j} t_{1}^{2} \in L$. If $i>2$ and $j<d-1$, proceeding as above, one will hit an element of the form $a_{1} a_{j} t_{1}^{2}, a_{1} a_{j} t_{1} t_{2}, a_{i} a_{d} t_{1} t_{2}$ or $a_{i} a_{d} t_{2}^{2}$, which will imply that $a_{i} a_{j} t_{1}^{2} \in L$. Similar arguments will give us the other required inclusions. Hence, $\operatorname{red}_{N}(M)=1$.

Corollary 4.3. Let $(R, \mathfrak{m})$ be a d-dimensional Cohen-Macaulay local ring, $I$ an $\mathfrak{m}$-primary ideal and $M=I \oplus \cdots \oplus I$ (r-times).

(i) If $d=2$, then $\operatorname{br}_{0}(M)-\mathrm{br}_{1}(M)=\ell(F / M)$ if and only if $\operatorname{red}(M)=1$.

(ii) If $d \geq 3, r=2$ and $\operatorname{br}_{0}(M)-\operatorname{br}_{1}(M)=\ell(F / M)$, then $\operatorname{red}(M)$ $=1$.

\section{Proof.}

(i) From Case 1 in the proof of Theorem 4.1, it follows that $\operatorname{br}_{0}(M)-$ $\operatorname{br}_{1}(M)=\ell(F / M)$ if and only if $e_{0}-e_{1}=\ell(R / I)$ if and only if $\operatorname{red}(I) \leq 1$ if and only if $\operatorname{red}(M)=1$, by Remark 2.4 .

(ii) From Case 2 in the proof of Theorem 4.1, it follows that $\operatorname{br}_{0}(M)-$ $\operatorname{br}_{1}(M)=\ell(F / M)$ if and only if $I$ is a parameter ideal. Now, it follows from Proposition 4.2 that, if $I$ is a parameter ideal, then $I \oplus I$ has reduction number one.

If the rank of $M$ is three, then an analogue Proposition 4.2 does not hold. Let $M=\mathfrak{m} \oplus \mathfrak{m} \oplus \mathfrak{m}$, where $\mathfrak{m}=(x, y, z) \subset k[[x, y, x]]$. Then it can be seen that the submodule $N$, generated by the columns of the 
matrix

$$
\left[\begin{array}{lllll}
x & y & z & 0 & 0 \\
0 & x & y & z & 0 \\
0 & 0 & x & y & z
\end{array}\right],
$$

is a minimal reduction of $M$ with $\operatorname{red}_{N}(M)=2$. The idea of getting minimal reduction of the above form comes from the work of Liu [12].

Example 4.4. Let $R=k[[X, Y]], I=\left(X^{3}, X^{2} Y^{4}, X Y^{5}, Y^{7}\right)$ and $J=\left(X^{3}, Y^{7}\right)$. Then $R$ is a two-dimensional regular local ring and $J$ is a minimal reduction of $I$ with reduction number 2 . It can be easily seen that

$$
P_{I}(n)=21\left(\begin{array}{c}
n+1 \\
2
\end{array}\right)-6\left(\begin{array}{l}
n \\
1
\end{array}\right)+1 .
$$

Set $F=R \oplus R, M=I \oplus I$. Then, again using [15, Theorem 2.5.2], we obtain $\mathrm{br}_{0}=63$ and $\mathrm{br}_{1}=33$. Therefore, $\operatorname{br}_{0}(M)-\mathrm{br}_{1}(M)=30<$ $32=\ell(F / M)$. Let $N$ be the submodule generated by the columns of

$$
\left[\begin{array}{ccc}
X^{3} & Y^{7} & 0 \\
0 & X^{3} & Y^{7}
\end{array}\right]
$$

Then, it can be seen that $N$ is a minimal reduction of $M$ with $\operatorname{red}_{N}(M)$ $=2$.

As in the case of ideals the example below shows that the CohenMacaulayness of the Rees algebra alone need not necessarily imply that $\operatorname{br}_{0}(M)-\operatorname{br}_{1}(M)=\ell(F / M)$ if $\operatorname{dim} R \geq 3$.

Example 4.5. Let $R=k[[X, Y, Z]], I=\left(X^{3}, X^{2} Y^{2}, Y^{3}, Z^{4}\right)$ and $M=I \oplus I$. It can be verified that $\mathcal{R}(M) \cong R\left[I t_{1}, I t_{2}\right]$ is CohenMacaulay. So, by [9, Theorem 6.1], $B F(n)=B P(n)$ for all $n \in \mathbb{N}$. The Buchsbaum-Rim polynomial can be computed as

$$
B P(n)=144\left(\begin{array}{c}
n+3 \\
4
\end{array}\right)-84\left(\begin{array}{c}
n+2 \\
3
\end{array}\right)+4\left(\begin{array}{c}
n+1 \\
2
\end{array}\right) .
$$

Therefore, $\operatorname{br}_{0}(M)-\operatorname{br}_{1}(M)=60<64=\ell(F / M)$.

We conclude the article with the following question. 
Question 4.6. Let $(R, \mathfrak{m})$ be a Cohen-Macaulay local ring of dimension $d>2$ and $M \subset F=R^{r}$ such that $\ell(F / M)<\infty$. Then is $\operatorname{br}_{0}(M)-$ $\operatorname{br}_{1}(M) \leq \ell(F / M)$ ? Does the equality $\operatorname{br}_{0}(M)-\operatorname{br}_{1}(M)=\ell(F / M)$ hold if and only if $\operatorname{red}_{N}(M)=1$ for some (any) minimal reduction $N$ of $M$ ?

Acknowledgments. The authors would like to thank E. Hyry, S. Zarzuela and W.V. Vasconcelos for going through a first draft and making many useful suggestions for improvement and further research. We are also thankful to the referee for a meticulous reading and suggesting several improvements.

\section{REFERENCES}

1. P.B. Bhattacharya, The Hilbert function of two ideals, Proc. Cambr. Philos. Soc. 53 (1957), 568-575.

2. J. Brennan, B. Ulrich and W.V. Vasconcelos, The Buchsbaum-Rim polynomial of a module, J. Algebra 241 (2001), 379-392.

3. D.A. Buchsbaum and D.S. Rim, A generalized Koszul complex II, Depth and multiplicity, Trans. Amer. Math. Soc. 111 (1964), 197-224.

4. F. Hayasaka and E. Hyry, A family of graded modules associated to a module, Comm. Algebra 36 2008, 4201-4217.

5. - On the Buchsbaum-Rim function of a parameter module, J. Algebra 327 (2011), 307-315.

6. C. Huneke, Hilbert functions and symbolic powers, Michigan Math. J. 34 (1987), 293-318.

7. C. Huneke and I. Swanson, Integral closure of ideals, rings, and modules, Lond. Math. Soc. Lect. Notes 336, Cambridge University Press, Cambridge, 2006.

8. E. Hyry, The diagonal subring and the Cohen-Macaulay property of a multigraded ring, Trans. Amer. Math. Soc. 351 (1999), 2213-2232.

9. , Cohen-Macaulay multi-Rees algebras, Comp. Math. 130 (2002), 319343.

10. A.V. Jayanthan and J.K. Verma, Grothendieck-Serre formula and bigraded Cohen-Macaulay Rees algebra, J. Algebra 254 (2002), 1-20.

11. D. Katz and V. Kodiyalam, Symmetric powers of complete modules over a two dimensional regular local ring, Trans. Amer Math. Soc. 349 (1997), 747-762.

12. J.-C. Liu, Rees algebras of finitely generated torsion free modules over a two dimensional regular local ring, Comm. Algebra 26 (1998), 4015-4039.

13. D.G. Northcott, $A$ note on the coefficients of the abstract Hilbert function, J. Lond. Math. Soc. 35 (1960), 209-214.

14. A. Ooishi, $\Delta$-genera and sectional genera of commutative rings, Hiroshima Math. J. 17 (1987), 361-372. 
15. P. Roberts, Multiplicities and Chern characters in local algebra, Cambridge University Press, New York, 1998.

16. A. Simis, B. Ulrich and W. Vasconcelos, Rees algebras of modules, Proc. Lond. Math. Soc. 87 (2003), 610-646.

17. W. Vasconcelos, Integral closure: Rees algebra, multiplicities, algorithms, Springer Monographs in Mathematics, Springer Verlag, New York, 2005.

18. J.K. Verma, Joint reductions of complete ideals, Nagoya Math. J. 118 (1990), 155-163.

Department of Mathematics, Indian Institute of Technology Madras, Chennai, 600036 India

Email address: r.balkrishnan@gmail.com

Department of Mathematics, Indian Institute of Technology Madras, Chennai, 600036 India

Email address: jayanav@iitm.ac.in 